\title{
A few recent developments in nano- photonics (Conference Presentation) (Withdrawal Notice)
}

\section{Marin Soljacic}

Marin Soljacic, "A few recent developments in nano-photonics (Conference Presentation) (Withdrawal Notice)," Proc. SPIE 11081, Active Photonic

Platforms XI, 110811X (29 October 2019); doi: 10.1117/12.2528895

Event: SPIE Nanoscience + Engineering, 2019, San Diego, California, United States 


\title{
A few recent developments in nano-photonics (Conference Presentation) (Withdrawal Notice)
}

\author{
Marin Soljacic \\ Massachusetts Institute of Technology (United States) \\ Proceedings Volume 11081, Active Photonic Platforms XI; 110811X (2019) \\ https://doi.org/10.1117/12.2528895
}

Event Active Photonic Platforms XI, 2019, San Diego, Califomia, United States

Online Publication Date: 9 September 2019

Withdrawn from Publication: 29 Oc to ber 2019

Publisher's Note: This conference presentation, originally published on 9 September 2019, was withdrawn per a uthor request. 